\title{
Antibióticos intratecales en pacientes adultos
}

\author{
ALBERTO FICA C. ${ }^{1}$
}

\author{
Intrathecal administration of antibiotics in adult patients
}

Intrathecal administration of antibiotics can be accomplished either by intralumbar or intraventricular route allowing a high and/or prolonged antibiotic concentration in the cerebrospinal fluid (CSF). Increase of ventriculitis rates associated to CNS shunts that involve multiresistant microorganisms has made relevant this route of administration due to the limited penetration of antibiotics active against multiresistant organisms through the blood brain barrier. Aminoglycosides and vancomycin are the most common antibiotics used for this purpose. In order to accomplish bactericidal activity and due to its concentration-dependent mechanism, aminoglycosides should reach concentrations 8 to 10 times above the MIC of a given Gram negative bacilli. Doses of 2 to $5 \mathrm{mg}$ for gentamicin and $30 \mathrm{mg}$ for amikacin every $24 \mathrm{~h}$ allow this purpose and has been associated to clinical cure in several reports. Vancomycin attains its bactericidal activity by a time-dependent mechanism and the best predictor of clinical efficacy is the time area under the curve (AUC) above the MIC. Doses of $20 \mathrm{mg}$ every day assure an adequate temporal exposition to the drug if trough concentrations of 5 to $10 \mu \mathrm{g} / \mathrm{ml}$ are obtained, and have been related to clinical efficacy. Intrathecal concentrations for these antibiotics are higher and therapeutically effective when they are administered through intraventricular route than by intralumbar puncture. Intraventricular antibiotics are indicated when a patient affected by ventriculitis does not improve despite maximal-doses systemic antibiotic therapy and/or CSF remains positive for microorganisms. In order to secure efficacy, intraventricular antibiotics should be implemented with removal of the ventricular drain associated to infection. Experience with quinuspristin/dalfopristin or linezolid for the treatment of staphylococcal ventriculitis is yet scarce.

Key words: Ventriculitis, Intratechal antimicrobials, Cerebrospinal fluid, Vancomycin, Aminoglycosides.

\section{Introducción}

Los antimicrobianos administrados por vía intratecal son necesarios en algunas infecciones del sistema nervioso central (SNC) para proveer, en un medio carente de mecanismos fagocíticos, concentraciones bactericidas de compuestos que tienen una baja penetración por la barrera hematoencefálica.

La administración intratecal considera la administración intralumbar o intraventricular de medicamentos y no reemplaza la terapia sistémica para controlar la infección fuera del SNC.

Los compuestos intratecales más utilizados corresponden a aminoglucósidos y vancomicina debido a la existencia y emergencia de infecciones asociadas a bacilos Gram negativos o cocáceas Gram positivas resistentes. Estos procesos in- cluyen meningitis o ventriculitis, en general de carácter nosocomial y asociadas a procedimientos neuroquirúrgicos y/o derivaciones ventriculares externas o internas. Las intervenciones quirúrgicas para efectuar un drenaje del LCR en pacientes con hidrocefalia hipertensiva se han expandido progresivamente en el país y las complicaciones infecciosas agregan una morbilidad o mortalidad considerable, de allí la relevancia de conocer esta forma terapéutica. Este documento trata especialmente de las ventriculitis e infecciones asociadas a drenajes ventriculares externos.

\section{Aspectos diagnósticos de las ventriculitis}

El diagnóstico de ventriculitis asociada a un drenaje ventricular no ha sido estandarizado. El espectro de alternativas incluye un cultivo del

\footnotetext{
1 Sección de Infectología. Departamento de Medicina, Hospital Clínico Universidad de Chile.
}

Recibido: 8 de enero 2003

Aceptado: 7 de mayo 2003 
LCR, más de un cultivo positivo o la asociación de cultivos positivos con pleocitosis e hipoglucorraquia (o aumento de proteínas) ${ }^{1}$. Algunos autores exigen además la existencia de fiebre a cambios en el estado mental y otros consideran la pleocitosis y la hipoglucorraquia en ausencia de cultivos positivos. Lozier et al, proponen discriminar entre sospecha de infección, infección asociada a un drenaje ventricular o ventriculostomía y ventriculitis propiamente tal (Tabla 1). Para estos autores, en presencia de un drenaje ventricular, la simple alteración de los niveles de glucosa, proteínas o un aumento en la celularidad del LCR, no es evidencia suficiente para asegurar la existencia de infección. La sospecha se establece cuando estos cambios son progresivos y los cultivos son negativos. La infección asociada a drenaje requiere que estos cambios progresivos se asocien a un resultado microbiológico positivo pero sin evidencias de síntomas neurológicos atribuibles. Finalmente, el diagnóstico de ventriculitis se establece ante la presencia de síntomas neurológicos, fiebre, y cambios citoquímicos en el LCR, aún cuando no exista confirmación microbiológica. También se ha propuesto utilizar determinaciones de lisozima $(>2,5 \mathrm{mg} / \mathrm{mL})$ y lactato (> 2,1 $\mathrm{mmol} / \mathrm{L})$ en el LCR para detectar casos de ventriculitis ${ }^{2}$.

No se puede establecer un valor absoluto de parámetros citoquímicos para indicar o sospechar infección, ya que algunas patologías provocan cambios evolutivos en las características del LCR. Por ejemplo, la hemorragia subaracnoidea se acompaña de hipoglucorraquia progresiva y un cultivo aislado puede indicar sólo contaminación. Tampoco se ha demostrado que el cultivo o estudio citoquímico rutinario del LCR a través del drenaje, permita identificar los casos de infección en forma más precoz que lo permitido por las manifestaciones clínicas y por ello no se recomienda su uso ${ }^{1,3}$.

\section{Agentes microbianos asociados a ventriculitis}

Diferentes autores señalan a las cocáceas Gram positivas como los agentes más frecuentes de infección asociada al drenaje ventricular ${ }^{1}$. El más importante de ellos corresponde a Staphylococcus epidermidis, secundado por Staphylococcus aureus. Los bacilos Gram negativos entéricos (por ejemplo Escherichia coli, Serratia sp o Enterobacter sp) o no fermentadores (Pseudomonas aeruginosa, Acinetobacter baumannii) son etiologías de importancia progresiva que han complicado las posibilidades terapéuticas por su estrecha asociación con multiresistencia ${ }^{4,5}$.

Los reportes sobre la presencia de diferentes
Tabla 1. Criterios para sospecha, infección asociada a ventriculostomía (drenaje ventricular externo) o ventriculitis (según Lozier et $\mathbf{a l}^{1}$ )

Sospecha de infección asociada a drenaje ventricular

- Hipoglucorraquia progresiva

- Aumento progresivo de valores de proteína en el LCR

- Aumento progresivo de pleocitosis

- Cultivos y tinciones negativas

Infección asociada a drenaje ventricular

- Hipoglucorraquia progresiva

- Aumento progresivo de valores de proteína en el LCR

- Aumento progresivo de pleocitosis

- Uno o más cultivos o tinción Gram de LCR positivos

- Ausencia de síntomas excepto fiebre

\section{Ventriculitis}

- Hipoglucorraquia

- Proteínas aumentadas en el LCR

- Pleocitosis

- Fiebre

- Signos de meningitis incluyendo rigidez de nuca, fotofobia, compromiso de conciencia o convulsiones

especies de cándida en el LCR son infrecuentes e incluyen C. albicans, $C$. parapsilosis y otras. $\mathrm{Su}$ relevancia clínica no es discutida cuando aparecen varios cultivos positivos para levaduras; sin embargo, un cultivo aislado probablemente sólo refleja una colonización y no una infección. Estos casos se asocian a valores de glucorraquia y celularidad normales en el LCR y ambas condiciones se pueden discriminar mediante una punción lumbar, ya que en casos de colonización, los cultivos del LCR obtenidos por vía lumbar son negativos ${ }^{6}$.

\section{Penetración de antibióticos al LCR}

El volumen de LCR alcanza aproximadamente a $130 \mathrm{ml}$ en pacientes adultos y se recambia 4 a 5 veces cada día. La penetración de los antimicrobianos administrados por vía sistémica depende fundamentalmente de su lipofilia o hidrofilia. Otros factores contribuyentes incluyen la magnitud de la inflamación meníngea, sistemas de transporte activo hacia el LCR, la unión a proteínas plasmáticas o el efecto de los corticoesteroides. Compuestos lipofílicos como quinolonas y rifampicina penetran fácilmente al LCR, en forma independiente al grado de inflamación meníngea. En contraste, la penetración de $\beta$-lactámicos y vancomicina depende de la existencia de una barrera hematoencefálica inflamada.

Los antimicrobianos no son metabolizados en el SNC y la concentración de los compuestos administrados por vía sistémica depende de su 
penetración y eliminación. Las concentraciones máximas no son alcanzadas al mismo tiempo que las logradas en el plasma, aunque la vida media de estos compuestos (ya sea si son aplicados vía sistémica o intraventricular) es, en general, superior a la observada en el plasma y ello permite intervalos de administración más prolongados. Se ha descrito un sistema de transporte activo para penicilina y ceftriaxona, de baja capacidad, que explica la obtención de concentraciones máximas en el LCR más rápido que lo permitiría su hidrofilia ${ }^{7}$.

A pesar de la entrada limitada de los $\beta$ lactámicos al LCR, la alta susceptibilidad de los patógenos comunitarios clásicos de meningitis bacteriana aguda (MBA), asegura que se sobrepase varias veces la CIM descrita para estos agentes ${ }^{8}$. Por ejemplo, para Haemophilus influenzae el rango de concentración descrito en LCR para ceftriaxona es de 2,1 a $7,2 \mu \mathrm{g} / \mathrm{mL}$, varias veces superior a los valores descritos de $\mathrm{CIM}_{90}$ para este fármaco $(0,01 \mu \mathrm{g} / \mathrm{mL})$. De la misma manera, el rango de concentraciones obtenidas con penicilina en el LCR es de 0,8 a 9,6 $\mu \mathrm{g} / \mathrm{mL}$, también superior al valor de $\mathrm{CIM}_{90}$ para cepas de Streptococcus pneumoniae susceptibles a este compuesto $(0,03 \mu \mathrm{g} / \mathrm{mL})$. El fenómeno es similar con Neisseria meningitidis, universalmente susceptible a penicilina. Concentraciones activas o útiles de antimicrobianos también se pueden lograr con terapia sistémica para algunos bacilos Gram negativos entéricos no resistentes tales como E. coli, con un valor de CIM $_{90} 0,25 \mu \mathrm{g} / \mathrm{mL}$ para cefotaxima o ceftriaxona ${ }^{9}$.

\section{Tratamiento de agentes resistentes por vía sistémica para bacilos Gram negativos y cocáceas Gram positivas}

La disponibilidad de quinolonas, cefalosporinas de cuarta generación (cefepime), carbapenémicos (meropenem), y combinaciones de $\beta$-lactámicos con inhibidores de $\beta$-lactamasas (sulbactam/ ampicilina), han permitido en algunos casos el tratamiento de infecciones del SNC por bacilos Gram negativos multiresistentes. Estos compuestos logran concentraciones apropiadas en el LCR para agentes comunitarios de MBA y han sido utilizados en ensayos comparativos con resultados similares a los obtenidos con cefalosporinas de tercera generación o con otros esquemas disponibles $^{9-11}$. Estudios randomizados con meropenem en altas dosis $(40 \mathrm{mg} / \mathrm{kg}$ dosis equivalentes a 2 g cada 8 h en adultos) han logrado concentraciones en el LCR entre 0,3 y $6,5 \mu \mathrm{g} / \mathrm{mL}$, superiores a las concentraciones obtenidas con dosis inferiores $(0,1$ a $2,8 \mu \mathrm{g} / \mathrm{mL}$ con dosis de 20 $\mathrm{mg} / \mathrm{kg}$ dosis $)^{12}$. En adultos sin meninges inflamadas, la concentraciones máximas de meropenem en el LCR han sido menores $(\sim 0,6 \mu \mathrm{g} / \mathrm{mL})$; sin embargo, las concentraciones obtenidas en el LCR con altas dosis de meropenem (con o sin meninges inflamadas), permiten asegurar la inhibición de las enterobacterias que se manifiestan susceptibles en el antibiograma para este compuesto $^{10,13}$. No obstante, estas concentraciones pueden ser marginales o claramente insuficientes para superar las $\mathrm{CIM}_{90}$ de meropenem contra P. aeruginosa $\left(\mathrm{CIM}_{90} \sim 3 \mu \mathrm{g} / \mathrm{mL}\right)$ o $A$. baumannii (4 a $6,4 \mu \mathrm{g} / \mathrm{mL}$ ), también involucrados como posibles agentes de ventriculitis ${ }^{10,14,15}$. Meropenem ha sido utilizado exitosamente en pacientes con ventriculitis por $P$. aeruginosa $a^{16}$, pero con selección de cepas resistentes in vivo en el manejo de pacientes con ventriculitis por $A$. baumannii ${ }^{17}$.

A pesar de que imipenem comparte las bondades antibacterianas asociadas a meropenem, no se considera una alternativa terapéutica en el tratamiento de las infecciones del SNC por su asociación ocasional con cuadros convulsivos en pacientes adultos con factores predisponentes que reciben altas dosis o en pacientes pediátricos previamente sanos ${ }^{18}$. Cefepime también ha sido asociado a neurotoxicidad con convulsiones y coma en pacientes que desarrollan insuficiencia renal sin ajuste de dosis ${ }^{19}$.

Este último compuesto, también puede ser considerado como una alternativa en el tratamiento de infecciones del SNC por especies bacterianas multiresistentes; posee una penetración al LCR semejante a las cefalosporinas de tercera generación y las concentraciones obtenidas en equilibrio antes de la siguiente dosis $(>1$ $\mu \mathrm{g} / \mathrm{mL}$ ) superan los valores de $\mathrm{CIM}_{90}$ de algunos agentes nosocomiales tales como E. coli CIM $_{90}$ $0,06 \mu \mathrm{g} / \mathrm{mL})$, Citrobacter freundii $\left(\mathrm{CIM}_{90} 0,5\right.$ $\mu \mathrm{g} / \mathrm{mL})$ o Enterobacter sp. $\left(\mathrm{CIM}_{90} 1 \mu \mathrm{g} / \mathrm{mL}\right)$. Sin embargo, estos valores resultan insuficientes para Klebsiella pneumoniae $\left(\mathrm{CIM}_{90} 2 \mu \mathrm{g} / \mathrm{mL}\right)$ o $P$. aeruginosa $\left(\mathrm{CIM}_{90} 32 \mu \mathrm{g} / \mathrm{mL}\right)^{20}$. Este compuesto ha sido utilizado exitosamente en algunos casos de infecciones por bacilos Gram negativos en altas dosis $(2 \mathrm{~g} \text { cada } 8 \mathrm{~h} \text { e.v. })^{21}$.

Ampicilina/sulbactam también ha sido utilizado en el tratamiento por vía sistémica de meningitis por A. baumannii multiresistentes o por agentes comunitarios ${ }^{11}$. La penetración de sulbactam al LCR es adecuada cuando éste es aplicado por vía endovenosa ${ }^{22}$ y menor cuando es aplicado por vía intramuscular o cuando no hay inflamación meníngea ${ }^{23,24}$. Las concentraciones de sulbactam/ampicilina deben sobrepasar los $4 \mathrm{y}$ $8 \mu \mathrm{g} / \mathrm{ml}$, respectivamente en el LCR, ya que 
estos valores representan el punto de corte para cepas susceptibles. Estudios en pacientes pediátricos con meningitis han permitido corroborar la penetración de ambos compuestos al LCR y la obtención de concentraciones útiles para agentes susceptibles ${ }^{23}$. La utilización de altas dosis (ampicilina $2 \mathrm{~g}$ - sulbactam $1 \mathrm{~g}$ cada 3 ó 6 horas por vía e.v.), ha permitido el tratamiento de pacientes con infecciones del SNC por $A$. baumannii, algunos de ellos asociados a ventriculitis o drenajes ventriculares ${ }^{25,26}$. Los valores de $\mathrm{CIM}_{90}$ de sulbactam sobre esta especie se aproximan a $8 \mu \mathrm{g} / \mathrm{mL}$, sin presentar este compuesto una actividad sinérgica con ampicilina, aunque ha sido utilizado exitosamente en infecciones no severas por este agente ${ }^{27}$. El efecto bactericida de sulbactam sobre $A$. baumannii es transitorio y resulta ser más pronunciado con concentraciones superiores a la CIM (4 x CIM), las que son difíciles de obtener en el LCR ${ }^{15}$; ello podría explicar resultados parciales obtenidos en el manejo de infecciones del SNC por esta especie ${ }^{25}$.

Las fluoroquinolonas tienen una buena penetración al LCR y no requieren dosis intratecales complementarias. Concentraciones bactericidas efectivas contra bacilos Gram negativos pueden lograrse con aumentos de las dosis parenterales a $400 \mathrm{mg}$ cada 8 horas. Estas dosis permiten concentraciones cercanas a $1 \mu \mathrm{g} / \mathrm{ml}$ en el $\mathrm{LCR}^{28}$.

La reciente disponibilidad de linezolid y de quinupristin/dalfopristin, antimicrobianos activos contra cocáceas Gram positivas resistentes a cloxacilina o vancomicina, podría facilitar el manejo de las ventriculitis asociadas a estos agentes ${ }^{29,30}$. A pesar de que teóricamente estos compuestos podrían reemplazar la necesidad de vancomicina intratecal y que se ha descrito su uso exitoso por vía intravenosa para el manejo de pacientes afectados por meningitis o ventriculitis, la experiencia con ambos compuestos es aún anecdótica y no han sido aprobados para infecciones del $\mathrm{SNC}^{31-33}$. Las concentraciones valle de linezolid obtenidas en el LCR luego de su administración endovenosa $(1,46$ a $7 \mu \mathrm{g} / \mathrm{mL})$ tienden a superar la $\mathrm{CIM}_{90}$ descrita para Staphylococcus spp resistentes o susceptibles a meticilina y Enterococcus faecium o E. faecalis $(2 \mu \mathrm{g} / \mathrm{mL})$; este compuesto ejerce un efecto sólo bacteriostático contra ellos y podría limitar el éxito terapéutico en este compartimiento ${ }^{34-36}$. Por otra parte, quinupristin/dalfopristin también carece de un efecto bactericida contra E. faecium, no es activo contra $E$. faecalis, requiere una vía central para su aplicación, está asociado a mialgias y artralgias en un número significativo de pacientes $(\sim 10 \%)$ y su penetración al LCR parece ser
Tabla 2. Alternativas de uso sistémico para el manejo de infecciones del SNC por agentes resistentes

En infecciones por bacilos Gram negativos resistentes* Ciprofloxacina en altas dosis (400 mg cada 8 horas) Meropenem en altas dosis ( $2 \mathrm{~g}$ cada 8 h e.v.)** Cefepime en altas dosis ( $2 \mathrm{~g}$ cada 8 h e.v. $)^{* * *}$ Sulbactam/ampicilina en altas dosis (2 g ampicilina-1 g sulbactam cada 3 a 6 h e.v.)****

\section{En infecciones por cocáceas Gram positivas resistentes*} Linezolid $600 \mathrm{mg}$ cada $12 \mathrm{~h}$ e.v.***** Quinupristin/dalfopristin $(7,5 \mathrm{mg} / \mathrm{kg} \text { cada } 8 \mathrm{~h} \text { e.v.) })^{* * * * * *}$

*: si el antibiograma revela susceptibilidad; **: no recomendado para bacilos Gram negativos no fermentadores; ***: no recomendado para bacilos Gram negativos no fermentadores y posiblemente en Klebsiella pneumoniae; ****: sólo efecto bacteriostático a las dosis señaladas contra A. baumannii; ***** experiencia anecdótica y de efecto bacteriostático contra cocáceas Gram positivas; ****** experiencia anecdótica, sin actividad contra $E$. faecalis y de efecto bacteriostático contra E. faecium, no disponible en Chile.

limitada ${ }^{29,30}$. Dosis de quinupristin/dalfopristin de 1 a $5 \mathrm{mg}$ al día por vía intratecal han sido utilizadas con éxito ocasional junto a otras terapias para el tratamiento de pacientes con meningitis causada por E. faecium resistente a vancomicina $^{33}$. Un resumen de las alternativas terapéuticas por vía sistémica para infecciones por agentes resistentes se presenta en la Tabla 2.

Los límites de la terapia sistémica con antimicrobianos están dictados por la existencia de infecciones del SNC provocadas por bacilos Gram negativos o por cocáceas Gram positivas resistentes a las alternativas por vía sistémica y por la baja penetración de los antimicrobianos aún activos para estos agentes. En estas condiciones, sólo la administración intratecal asegura concentraciones terapéuticas adecuadas. La emergencia de infecciones nosocomiales del SNC por bacilos Gram negativos entéricos o no fermentadores e integrantes del género Staphylococcus resistentes, ha hecho relevante el conocimiento y manejo de la administración intratecal de antimicrobianos. Los compuestos propios de este escenario corresponden a gentamicina, amikacina y vancomicina. La resistencia de los bacilos Gram negativos a cloranfenicol o ampicilina, compuestos que tienen una buena penetración, impide su uso actualmente.

\section{Aspectos farmacocinéticos y farmacodinámicos}

Los principios terapéuticos de las infecciones del SNC señalan que la eficacia está relacionada 
con la obtención de concentraciones bactericidas apropiadas en el sitio de infección, ya que la inhibición bacteriana es insuficiente en un ambiente con mecanismos fagocíticos ausentes o a lo más, presentes en forma transitoria. Sin embargo, el efecto bactericida de un antimicrobiano no se logra de la misma forma para todos los antimicrobianos. Dos grandes modelos de acción han emergido sobre el efecto bactericida y la eficacia terapéutica. Uno de ellos, propio de los $\beta$-lactámicos, relaciona el efecto bactericida al tiempo en que la concentración del compuesto está sobre la CIM entre 2 dosis ( $\mathrm{T}>\mathrm{CIM}$ ). Para un efecto apropiado y dependiendo del agente y antimicrobiano específico, estos fármacos deben estar sobre la CIM al menos 40 a $60 \%$ del intervalo de tiempo entre 2 dosis $7,37,38$. Los carbapenémicos requieren menos tiempo sobre la CIM que las penicilinas ( $\mathrm{T}>\mathrm{CIM} \sim 40 \%$ para estos últimos) y éstas a su vez menos tiempo que las cefalosporinas ( $\mathrm{T}>\mathrm{CIM} \sim 50 \%$ ) para asegurar un efecto bactericida y una eficacia terapéuti$\mathrm{ca}^{38}$. El otro modelo, denominado concentracióndependiente, indica que el efecto bactericida está determinado por la cantidad de veces que la concentración máxima o pico del antimicrobiano sobrepasa la CIM (C pico/CIM). Este modelo es propio de los aminoglucósidos y de las fluoroquinolonas $^{38}$. Sin embargo, las fluoroquinolonas evidencian un comportamiento mixto en las infecciones del SNC con un patrón tiempo-dependiente y también concentración-dependiente ${ }^{7}$. Vancomicina no tiene un efecto bactericida concentración-dependiente, se comporta bajo un modelo tiempo-dependiente y el mejor predictor de su eficacia terapéutica es el tiempo en que el área bajo la curva se encuentra sobre la CIM (AUC > (IM) ${ }^{38}$.

Aminoglucósidos. Como se ha mencionado, el efecto bactericida de los aminoglucósidos es dependiente de la concentración del antimicrobiano y ella debe sobrepasar la CIM 8 a 10 veces para asegurar un efecto bactericida adecuado. Este principio es pivotal para lograr la erradicación microbiológica y permitir la curación. El valor de CIM ante gentamicina para cepas susceptibles de bacilos Gram negativos, incluyendo $P$. aeruginosa, es de $4 \leq \mu \mathrm{g} / \mathrm{mL}$ y para amikacina, de $16 \mu \mathrm{g} / \mathrm{mL}$. Las cepas susceptibles de estas especies tienen en general cifras de CIM muy inferiores a estos límites.

La concentración de estos compuestos luego de la administración intratecal debe permitir alcanzar $\geq 8-10 \times$ [CIM] en el LCR $(\geq 32-40 \mu \mathrm{g}$ / $\mathrm{mL}$ para gentamicina $\mathrm{y} \geq 128-160 \mu \mathrm{g} / \mathrm{mL}$ para amikacina $)^{39,40}$. El efecto bactericida no es tiempo- dependiente en los aminoglucósidos y sólo es necesario alcanzar transitoriamente estas concentraciones elevadas.

La aplicación de una dosis ventricular de 10 mg de gentamicina ha permitido alcanzar concentraciones máximas de $80 \mu \mathrm{g} / \mathrm{mL}$ y dosis de 2,5 $\mathrm{mg}$ han estado asociadas a concentraciones máximas entre 10 a $130 \mu \mathrm{g} / \mathrm{mL}^{30,41}$. La dosis intraventricular recomendada de gentamicina es de 2 a $5 \mathrm{mg}$ por día. En el caso de amikacina, la dosis intraventricular recomendada es de $30 \mathrm{mg}$ al día o $0,3 \mu \mathrm{g} / \mathrm{mL}$ estimado de LCR. Estas dosis han permitido alcanzar concentraciones superiores a los $140 \mu \mathrm{g} / \mathrm{mL}$ en el LCR ${ }^{42}$. En contraste, la terapia sistémica con estos compuestos no permite alcanzar concentraciones bactericidas en el LCR $(\leq 3 \mu \mathrm{g} / \mathrm{mL}$ para gentamicina $0 \leq 6 \mu \mathrm{g} / \mathrm{mL}$ de amikacina).

Diferentes series de casos han demostrado la eficacia terapéutica de gentamicina o amikacina intratecal para el tratamiento de infecciones por agentes susceptibles y la seguridad y baja toxicidad de este antimicrobiano ${ }^{40-45}$. A pesar de que el único estudio randomizado disponible para este antimicrobiano (desarrollado con lactantes) no logró demostrar un efecto positivo para gentamicina, la literatura y evidencia respalda mayoritariamente su uso, debido a los sesgos descritos en el trabajo original (ver más adelante $)^{46-49}$. La relación establecida entre un aumento de los niveles de endotoxina e interleukina-1b y un pronóstico desfavorable en aquellos pacientes lactantes que recibieron terapia intraventricular con gentamicina, también esta influenciada por estos sesgos ya que fueron elaborados con una fracción de los pacientes incluidos en el estudio criticado ${ }^{50}$.

Vancomicina. Las concentraciones de vancomicina en el LCR no superan en general, 5 a $6 \mu \mathrm{g} /$ $\mathrm{mL}$, luego de la administración parenteral de este compuesto. En contraste, las concentraciones alcanzadas con una dosis intratecal de $20 \mathrm{mg}$ llegan en promedio a los 200 a $300 \mu \mathrm{g} / \mathrm{mL}$ de LCR y niveles valle de $20 \mu \mathrm{g} / \mathrm{mL}$. Estas concentraciones son superiores a los valores de $\mathrm{CIM}_{90}$ descritos para cepas estafilocóccicas resistentes a cloxacilina o meticilina. Para $S$. aureus resistentes a cloxacilina esta cifra es $1 \mu \mathrm{g} / \mathrm{mL}$ y para Staphylococcus coagulasa negativa también resistentes, esta cifra es de $2 \mu \mathrm{g} / \mathrm{mL}$. Aproximadamente $20 \%$ de las cepas del género Staphylococcus son tolerantes a vancomicina, es decir presentan una gran diferencia entre la concentración bactericida mínima (CBM; hasta $16 \mu \mathrm{g} / \mathrm{mL})$ y la $\mathrm{CIM}^{51}$.

El efecto bactericida de vancomicina está asociado a la exposición mantenida del compuesto 
sobre las cepas susceptibles, y concentraciones antes de la siguiente dosis de 5 a $10 \mu \mathrm{g} / \mathrm{mL}$ implican que se ha logrado esta exposición y un valor adecuado de AUC $>\mathrm{CIM}^{52}$. En este sentido, el efecto bactericida de vancomicina es tiempodependiente y no se incrementa al aumentar las dosis y concentración máxima en forma local. El efecto bactericida de vancomicina no es modificado por la presencia de proteínas en el sitio de infección. Sin embargo, la presencia de biopolímeros (slime), como ocurre por ejemplo en la superficie de un drenaje ventricular externo colonizado por Staphylococcus coagulasa negativa, incrementa la $\mathrm{CBM}$ en varias diluciones aunque no modifica la CIM. En estas condiciones es fácil lograr una inhibición bacteriana pero no la esterilidad del LCR, a no ser que se remueva el cuerpo extraño. El efecto bactericida de vancomicina se obtiene lentamente y se requieren $\sim 48$ horas para lograr una reducción significativa de una población bacteriana determinada ${ }^{52}$.

En un paciente pediátrico con alergia a vancomicina se utilizó exitosamente teicoplanina sistémica e intratecal $(10 \mathrm{mg})$ para el manejo de ventriculitis por Staphylococcus coagulasa negativa $^{53}$. Sin embargo, teicoplanina es menos activa que vancomicina contra aislamientos de Staphylococcus coagulasa negativa $\left(\mathrm{CIM}_{90}\right.$ de 1 a $12 \mu \mathrm{g} / \mathrm{mL}$ con teicoplanina versus valores de 1 a $4 \mu \mathrm{g} / \mathrm{mL}$ para vancomicina). Lo opuesto ocurre con $S$. aureus. Es probable que en adultos se requieran dosis intraventriculares > a $10 \mathrm{mg}$ (similares a vancomicina) en caso de plantearse su uso contra diferentes especies estafilocóccicas, en parte por la menor actividad contra el grupo coagulasa negativa, porque teicoplanina tiene un menor efecto bactericida que vancomicina y porque su efecto disminuye en presencia de proteínas ${ }^{54}$. Al igual que para los aminoglucósidos, diferentes series de casos demuestran la eficacia terapéutica del uso de vancomicina intratecal ${ }^{55,56}$.

Cefalosporinas, anfotericina $B$ y otros antimicrobianos. La aplicación de $\beta$-lactámicos intratecales ha sido relegada a un nivel histórico por la disponibilidad de compuestos similares con buena penetración al LCR (por ejemplo cefotaxima, ceftriaxona o cefepime) y por la toxicidad asociada al uso intratecal de ellos. Las manifestaciones adversas del uso intratecal incluyen paraplejia, hemiplejia, radiculitis o convulsiones y han sido observadas ante penicilina, cefazolina y otros $\beta$ lactámicos ${ }^{39}$. De la misma manera, el uso intratecal de anfotericina $\mathrm{B}$ no es necesario en la mayor parte de las infecciones fúngicas debido a los resultados obtenidos con la terapia sistémica con este compuesto ${ }^{57}$, a la disponibilidad de antimi- cóticos alternativos como fluconazol o voricona$\mathrm{zol}^{58,59}$ y a la descripción de efectos adversos mayores con el uso intratecal, tales como mielopatía, delirio, parkinsonismo, radiculitis y alteraciones visuales $^{39}$. En casos de mala respuesta al tratamiento sistémico se puede utilizar una dosis intratecal diaria de 0,1 a $0,5 \mathrm{mg}$ por aproximadamente 10 días, en el caso de infecciones por Candida sp asociadas a un drenaje ventricular ${ }^{39}$. Las fluoroquinolonas evidencian un comportamiento mixto del efecto bactericida en las infecciones del SNC por lo que se debe asegurar una dosis efectiva?

\section{Variaciones en la concentración local de antimicrobianos en el LCR}

El LCR es un ultrafiltrado del plasma, producido en los plexos coroideos y que circula en un sentido céfalo-caudal. Esta dirección determina varios aspectos que modifican la concentración local de antimicrobianos, de acuerdo a su lugar de administración:

- La concentración de un antimicrobiano en los ventrículos cerebrales es mayor cuando el compuesto se administra directamente en ellos. En contraste, su concentración es notoriamente menor cuando el compuesto se aplica por vía intralumbar. Por ejemplo se ha señalado que la concentración promedio en ventrículos alcanza un rango de 0,0 a $2,1 \mu \mathrm{g} / \mathrm{mL}$ con la administración intralumbar versus cifras de 12 a $40 \mu \mathrm{g} / \mathrm{mL}$ con la administración intraventricular directa de gentamicina ${ }^{41}$.

- La llegada de antimicrobianos hacia caudal está limitada desde el territorio ventricular en aquellos casos donde hay obstrucciones al flujo del LCR.

- La presencia de hidrocefalia aumenta el volumen de distribución y disminuye la concentración local del antimicrobiano. La magnitud de esta disminución no es importante siempre y cuando se utilicen dosis apropiadas ${ }^{60}$.

- La existencia de limitaciones al flujo céfalocaudal del LCR prolonga la vida media de los compuestos administrados por vía intratecal en el compartimiento donde fueron administrados.

- La existencia de un drenaje ventricular externo facilita la eliminación de un antimicrobiano determinado, disminuyendo su vida media.

\section{La importancia de la remoción del drenaje ventricular}

La presencia de un drenaje ventricular externo o interno asociado especialmente a una infección por S. epidermidis, Candida sp o Pseudomonas 
sp, permite la formación de una biopelícula que aumenta notoriamente la CBM del microorganismo y limita la esterilización del LCR ${ }^{61}$. En estas circunstancias, la remoción del catéter de drenaje asegura la curación de la infección. La tasa de curación puede superar el $90 \%$ cuando se considera el retiro del catéter de drenaje en el tratamiento versus cifras inferiores a $50 \%$ cuando éste permanece in situ ${ }^{55}$.

En el caso de infecciones por levaduras, la remoción del catéter o drenaje ventricular es fundamental para asegurar las posibilidades de una terapia eficaz. El género Candida, también está asociado a la síntesis de una película de biopolímero que antagoniza el efecto fungistático o fungicida de diferentes compuestos. A pesar de que la terapia sistémica con anfotericina B está asociada a una baja penetración al $\mathrm{LCR}^{39}$, los reportes indican que una remoción del catéter asociada a una terapia sistémica con este compuesto, ha permitido curar una fracción importante de los pacientes afectados ${ }^{62}$.

\section{Toxicidad}

La información sobre la toxicidad de los aminoglucósidos intraventriculares es discordante. Los reportes derivados del estudio cooperativo sobre meningitis neonatal que señalaban una evolución adversa con mayor letalidad asociada a gentamicina intraventricular en comparación a gentamicina más ampicilina sistémica en el manejo de pacientes con meningitis neonatal, han sido criticados por la multiplicidad de sesgos en el diseño del trabajo en desmedro del grupo que recibió terapia intratecal. En efecto, se critica que los pacientes con gentamicina intratecal iniciaron su tratamiento en forma más tardía, presentaron una mayor frecuencia de bacilos resistentes a gentamicina, alcanzaron a recibir un escaso número de dosis por diferencias en la severidad inicial del cuadro, se incluyeron muertes no relacionadas al uso de antimicrobianos en el grupo intratecal y se efectuaron más cambios de tratamiento no especificados en el grupo sin terapia intratecal ${ }^{46,47,49,63}$. Por otra parte, la gentamicina era aplicada con punciones repetidas sobre los ventrículos, una forma de administración que aporta un riesgo intrínseco al procedimiento y que no es utilizada actualmente por el riesgo de porencefalia. Esta información es diferente a la obtenida con numerosos otros estudios que señalan que los aminoglucósidos administrados por vía intratecal se asocian en general a escasos efectos adversos y que se consideran compuestos relativamente seguros ${ }^{39,42,44,60}$. En particular no están asociados a nefrotoxicidad cuando son utilizados en forma intratecal aislada y los posibles efectos ototóxicos han sido inconstantes y sólo observados en algunos reportes. No obstante, la administración de gentamicina intralumbar ha provocado en ocasiones dolor radicular $\mathrm{y}$ aracnoiditis ${ }^{39}$.

De la misma manera, la experiencia señala que el uso de vancomicina es, en general, bastante seguro y sólo existe un reporte aislado de confusión mental transitoria ${ }^{39,55}$.

\section{Forma de administración y duración del tratamiento}

Los antimicrobianos intratecales deben ser administrados diluidos en solución salina al $9 \%$ o estéril y sin preservantes. El volumen habitual a utilizar es de 2 ó $5 \mathrm{~mL}$ y se debe remover inicialmente un volumen similar antes de colocar la dosis, para evitar aumentos de la presión intracerebral. Para asegurar la entrada del antimicrobiano, la dosis administrada puede ser seguida de un volumen equivalente de solución salina (por ejemplo $2 \mathrm{ml}$ de solución con antimicrobiano seguido de $2 \mathrm{ml}$ de solución salina). Parte del volumen extraído puede ser enviado a cultivo y/o estudio citoquímico, teniendo cuidado de no enviar los primeros $2 \mathrm{ml}$ de muestra que representan el espacio muerto del drenaje. Para asegurar la distribución adecuada del antimicrobiano en todo el LCR, se debe interrumpir el circuito por 1 a 3 horas aproximadamente ${ }^{56,64}$. Los antimicrobianos intratecales han sido mayoritariamente usados hasta obtener una mejoría clínica y varios cultivos negativos de LCR, aunque la duración precisa del tratamiento no ha sido establecida. Las dosis habituales y las diluciones para los tres antimicrobianos más usados se señalan en la Tabla 3.

\section{Indicaciones potenciales de antimicrobianos intratecales}

El uso de antimicrobianos intratecales no es imperativo en todas las infecciones del SNC, incluso aquellas asociadas a agentes resistentes. Su uso está claramente indicado en aquellos casos con infección asociada a un drenaje o ventriculitis donde no existen alternativas sistémicas disponibles y donde a pesar de la terapia sistémica no se ha logrado una mejoría del paciente o una esterilización del LCR. Otras indicaciones potenciales incluyen el tratamiento de una infección asociada a un drenaje ventricular cuando no es posible plantear su retiro o reemplazo. La eficacia del uso profiláctico de antimicrobianos intratecales en presencia de un drenaje ventricular, no ha sido claramente demostrado ${ }^{65,66}$. Un resumen de los 
Tabla 3. Dosis y diluciones de antimicrobianos de uso intraventricular

\begin{tabular}{ccc}
\hline Compuesto & Indicaciones potenciales (ver texto) & Dosis habitual ; dilución* \\
\hline Gentamicina & $\begin{array}{l}\text { Infecciones por bacilos Gram } \\
\text { negativos resistentes }\end{array}$ & $2-5 \mathrm{mg}$ cada $24 \mathrm{~h} ; 2 \mathrm{mg} / \mathrm{mL}$ \\
Amikacina & Idem & $30 \mathrm{mg} \mathrm{cada} 24 \mathrm{~h} ; 10 \mathrm{mg} / \mathrm{mL}$ \\
Vancomicina & $\begin{array}{l}\text { Infecciones por estafilococos } \\
\text { resistentes a cloxacilina }\end{array}$ & $20 \mathrm{mg} \mathrm{cada} 24 \mathrm{~h} ; 10 \mathrm{mg} / \mathrm{mL}$ \\
\hline
\end{tabular}

* las diluciones en solución salina al 9\%o deben prepararse para un volumen final a instilar de 2 a $5 \mathrm{~mL}$.

Tabla 4. Conclusiones sobre el uso de antimicrobianos intratecales

- Los antimicrobianos intratecales son una alternativa terapéutica en aquellos pacientes afectados por infecciones del SNC por agentes resistentes y en los cuales existe una baja penetración de antibióticos activos a través de la barrera hematoencefálica

- Antes de ser indicados se debe analizar si el caso es susceptible de ser tratado con cefalosporinas de tercera o cuarta generación o meropenem por vía sistémica.

- Sulbactam/ampicilina puede ser considerado como una alternativa terapéutica por vía sistémica contra A. baumannii, sin embargo, su efecto con las concentraciones alcanzadas en el LCR es sólo bacteriostático.

- Quinupristin/dalfopristin y linezolid también podrían ser considerados para una terapia sistémica para el tratamiento de infecciones por cocáceas Gram positivas resistentes. Sin embargo, la experiencia con estos productos es escasa. Linezolid ejerce un efecto bacteriostático contra estas bacterias al igual que quinupristin/dalfopristin contra E. faecium. Este último además no es activo contra E. faecalis.

- Las indicaciones potenciales de antimicrobianos intraventriculares incluyen aquellos pacientes con infecciones del SNC (infección o ventriculitis) por alguna de las siguientes situaciones: bacilos Gram negativos resistentes a cefalosporinas de tercera o cuarta generación y a meropenem o cocáceas Gram positivas resistentes a $\beta$-lactámicos; pacientes sin una respuesta apropiada a un tratamiento sistémico con un fármaco activo; pacientes en los que no se ha conseguido esterilizar el LCR a pesar de una terapia sistémica prolongada. Potencialmente podrían considerarse en todos aquellos casos donde no se pueda retirar o reemplazar el catéter de drenaje ventricular.

- En la mayor parte de los casos, estos antimicrobianos son: gentamicina, amikacina y vancomicina

- Las dosis de estos antimicrobianos deben ser apropiadas para optimizar su efecto bactericida. La vida media prolongada de ellos en el LCR permite una administración diaria

- Las mayores concentraciones de estos antimicrobianos se obtiene al usar la vía intraventricular en comparación a la aplicación intralumbar. Cada vez que sea posible se debe utilizar esta vía.

- La aplicación intraventricular de antimicrobianos se puede efectuar mediante un drenaje ventricular externo en uso o mediante un reservorio.

- No hay información clara sobre la duración de este tratamiento, aunque habitualmente se efectúa hasta obtener unos 3 ó 4 cultivos consecutivos negativos de LCR. Tampoco existe claridad sobre la necesidad de una terapia sistémica asociada en ausencia de infección diseminada.

- Cada vez que sea posible, se debe plantear el retiro y/o reemplazo del drenaje ventricular involucrado en la infección para asegurar la eficacia terapéutica.

aspectos comentados en esta publicación se entrega en la Tabla 4.

\section{Referencias}

1.- Lozier A P, Sciacca R R, Romagnoli M F, Connoly E S Jr. Ventriculostomy-related infections: a critical review of the literature. Neurosurg 2002; 51: 170-82.

2.- Schroeder S, Stuerenburg H J, Escherich F, Pfeiffer G. Lysozyme in ventriculitis: a marker for diagnosis and disease progression. J Neurol Neurosurg Psychiatry
2000; 69: 696-7.

3.- Hader W J, Steinbock P. The value of routine cultures of the cerebrospinal fluid in patients with external ventricular drains. Neurosurgery 2000; 46: 1149-55.

4.- Lyke K E, Obasanjo O O, Williams M A, O’Brien M, Chotani R, Perl T M. Ventriculitis complicating use of intraventricular catheters in adult neurosurgical patients. Clin Infect Dis 2001; 33: 2028-33.

5.- Siegman-Igra Y, Bar-Yosef S, Gorea A, Avram J. Nosocomial Acinetobacter meningitis secondary to invasive procedures: Report of 25 cases and review. Clin Infect Dis 1993; 17: 843-9. 
6.- Geers T A, Gordon S M. Clinical significance of Candida species isolated from cerebrospinal fluid following neurosurgery. Clin Infect Dis 1999; 28: 1139-47.

7.- Lutzar I, McCracken G H, Friedland I R. Antibiotic pharmacodynamics in cerebrospinal fluid. Clin Infect Dis 1998; 27: 1117-29.

8.- Lutzar I, Friedland I R. Pharmacokinetics and pharmacodynamics of cephalosporins in cerebrospinal fluid. Clin Pharmacokinetics 2000; 39: 335-43.

9.- Andes D R, Craig W A. Pharmacokinetics and pharmacodynamics of antibiotics in meningitis. Infect Dis Clin North Am 1999; 13: 595-618.

10.- Wiseman L R, Wagstaff A J, Brogden R N, Bryson H R. Meropenen. A review of its antibacterial activity, pharmacokinetic properties and clinical efficacy. Drugs 1995; 50: 73-101.

11.- Rodríguez W J, Khan W N, Puig J et al. Sulbactam/ ampicillin vs. chloramphenicol/ampicillin for the treatment of meningitis in infants and children. Rev Infect Dis 1986; 8 Suppl 5: S620-9.

12.- Dagan R, Velghe L, Rodda J L, Klugman K P. Penetration of meropenem into the cerebrospinal fluid of patients with inflammed meninges. J Antimicrob Chemother 1994; 34: 175-79.

13.- Nau R, Lassek C, Kinzig-Schippers M, Thiel A, Prange $\mathrm{H} \mathrm{W}$, Sorgel F. Disposition and elimination of meropenem in cerebrospinal fluid of hydrocephalic patients with external ventriculostomy. Antimicrob Agents Chemother 1998; 42: 2012-16.

14.- Triantafilo V, Fica A, Silva M, Thompson L. E-test para determinar concentraciones inhibitorias mínimas, estimar la diversidad bacteriana e identificar presuntivamente beta-lactamasas en cepas de Pseudomonas aeruginosa y Acinetobacter baumannii asociadas a infecciones intrahospitalarias. Rev Méd Chile 1997; 125: $149-60$

15.- Appleman M D, Belzberg H, Citron D M et al. In vitro activities of nontraditional antimicrobial against multiresistant Acinetobacter baumannii strains isolated in an intensive care unit outbreak. Antimicrob Agents Chemother 2000; 44: 1035-40.

16.- Barberán J, García M C, Campos A, Khorrami S. Meningitis por Pseudomonas aeruginosa. Tratamiento con meropenem. Enferm Infecc Microbiol Clin 1998; 16: 249

17.- Núñez M L, Martínez-Toldos M C, Bru M, Simarro E, Segovia M, Ruiz J. Appearance of resistance to meropenem during the treatment of a patient with meningitis by Acinetobacter. Scand J Infect Dis 1998; 30: 421-3.

18.- Buckley M M, Brogden R N, Barradell L B, Goa K L. Imipenem/Cilastatin. A reappraisal of its antibacterial activity, pharmacokinetic properties and therapeutic efficacy. Drugs 1992; 44: 408-44.

19.- Chatellier D, Jourdain M, Mangalaboyi J et al. Cefepime-induced neurotoxicity: an underestimated complication of antibiotherapy in patients with acute renal failure. Intensive Care Med 2002; 28: 14-217.

20.- Rhoney D H, Tam V H, Parker D Jr, McKinnon P S, Coplin W M. Disposition of cefepime in the central nervous system of patients with external ventricular drains. Pharmacotherapy 2003; 23: 310-4.

21.- Barnes B J, Wiederhold N P, Micek S T, Polish L B, Ritchie D J. Enterobacter cloacae ventriculitis successfully treated with cefepime and gentamicin: case report and review of the literature. Pharmacotherapy 2003; 23(4): 537-42.
22.- Stahl J P, Bru J P, Fredj G, Brammer K W, Malleret M R, Micoud M. Penetration of sulbactam into the cerebrospinal fluid of patients with bacterial meningitis receiving ampicillin therapy. Rev Infect Dis 1986; 8 Suppl 5: S612-6.

23.- Foulds G, McBride T J, Knirsch AK, Rodríguez W J, Khan W N. Penetration of sulbactam and ampicillin into cerebrospinal fluid of infants and young children with meningitis. Antimicrob Agents Chemother 1987; 31: $1703-5$.

24.- Hanninen P, Rossi T. Penetration of sulbactam into cerebrospinal fluid of patients with viral meningitis or without meningitis. Rev Infect Dis 1986; 8 Suppl: S609-11.

25.- Jiménez-Mejías M E, Pachon J, Becerril B, PalominoNicas J, Rodríguez-Cobacho A, Revuelta M. Treatment of multidrug-resistant Acinetobacter baumannii meningitis with ampicillin/sulbactam. Clin Infect Dis 1997; 24: $932-5$.

26.- Cawley M J, Suh C, Lee S, Ackerman B H. Nontraditional dosing of ampicillin-sulbactam for multidrug-resistant Acinetobacter baumannii meningitis. Pharmacotherapy 2002; 22 (4): 527-32.

27.- Corbella X, Ariza J, Ardanuy C et al. Efficacy of sulbactam alone and in a combination with ampicillin in nosocomial infections caused by multiresistant Acinetobacter baumannii. J Antimicrob Chemother 1998; 42: 793-802.

28.- Lipman J, Allworth A, Wallis S C. Cerebrospinal fluid penetration of high doses of intravenous ciprofloxacin in meningitis. Clin Infect Dis 2000; 31: 1131-3.

29.- Linden P K. Treatment options for vancomycinresistant enterococal infections. Drugs 2002; 62: 42541.

30.- Livermore D M. Quinupristin/dalfopristin and linezolid: where, when, which and whether to use? J Antimicrob Chemother 2000; 46: 347-50.

31.- Garey K W, Tesoro E, Muggia V, Pasquier O, Rodvold K A. Cerebrospinal fluid concentration of quinupristindalfopristin in a patient with vancomycin-resistant Enterococcus faecium ventriculitis. Pharmacotherapy 2001; 21: 748-50.

32.- Graham P L, Ampofo K, Saiman L. Linezolid treatment of vancomycin-resistant Enterococcus faecium ventriculitis. Pediatr Infect Dis J 2002; 21: 798-800.

33.- Zeana C, Kubin CJ, Della-Latta P, Hammer S M. Vancomycin-resistant Enterococcus faecium meningitis successfully managed with linezolid: case report and review of the literature. Clin Infect Dis 2001; 33: 477-82.

34.- Wise R, Andrews J M, Boswell F J, Ashby J P. The invitro activity of linezolid (U-100766) and tentative breakpoints. J Antimicrob Chemother 1998; 42: 7218.

35.- Villani P, Regazzi M B, Viale P et al. Cerebrospinal fluid linezolid concentrations in postneurosurgical central nervous system infections. Antimicrob Agents Chemother 2002; 46: 936-7.

36.- Perry C M, Jarvis B. Linezolid. A review of its use in the management of serious Gram-positive infections. Drugs 2001; 61: 525-51.

37.- Labarca J. Nuevos conceptos en farmacodinamia ¿Debemos repensar como administramos antimicrobianos? Rev Chil Infect 2002; 19 (Supl 1): S28-S32.

38.- Craig W A. Pharmacokinetic/pharmacodynamic parameters: rationale for antibacterial dosing of mice and men. Clin Infect Dis 1998; 26: 1-12. 
39.- Wen D Y, Bottini A G, Hall W A, Haines S J. The intraventricular use of antibiotics. Neurosurg Clin $\mathrm{N}$ Am 1992; 3: 343-54.

40.- Wald S L, McLaurin R L. Cerebrospinal fluid antibiotic levels during treatment of shunt infections. J Neurosurg 1980; 52: 41-6.

41.- Kaiser A B, McGee Z A. Aminoglycoside therapy of Gram negative bacillary meningitis. $N$ Engl J Med 1975; 293: 1215-20.

42.- Wirt T C, McGee ZA, Oldfield E H, Meacham W F. Intraventricular administration of amikacin for complicated Gram-negative meningitis and ventriculitis. J Neurosurg 1979; 50: 95-9.

43.- Moellering R C Jr, Fischer E G. Relationship of intraventricular gentamicin levels to cure of meningitis. J Pediatr 1972; 81: 534-7.

44.- Rahal J J, Hyams P J, Simberkoff M S, Rubinstein E. Combined intrathecal and intramuscular gentamicin for Gram-negative meningitis. N Engl J Med 1974; 290: 1394-8.

45.- Young R F, Yoshimori R N, Murray D L, Chou P J. Postoperative neurosurgical infections due to Bacillus species. Surg Neurol 1982; 18: 271-3.

46.- McCracken G H Jr, Mize S G, Threlkeld N. Intraventricular gentamicin therapy in Gram-negative bacillary meningitis of infancy. Report of the second neonatal meningitis cooperative study group. Lancet 1980; I: 787-91.

47.- Corbeel L, De Boeck K, Logghe $\mathrm{N}$ et al. Intraventricular gentamicin in meningitis (letter). Lancet 1980; 2 (8188): 252-3.

48.- Swartz M N. Intraventricular use of aminoglycosides in the treatment of Gram-negative bacillary meningitis: Conflicting views. J Infect Dis 1981; 143: 293-6

49.- Warren R E, Roberton N R C. Intraventricular gentamicin in meningitis (Letter). Lancet 1980; 2 (8188): 252.

50.- Mustafa M M, Mertsola J, Ramilo O, Saez-Llorens X, Risser R C, McCracken G H Jr. Increased endotoxin and interleukin $1 \mathrm{~b}$-concentrations in cerebrospinal fluid of infants with coliform meningitis and ventriculitis associated with intraventricular gentamicin therapy. J Infect Dis 1989; 160: 891-5.

51.- Fekety R. Vancomycin and teicoplanin. En: Mandell, Douglas and Bennett's Principles and Practice of Infectious Diseases. Mandell GL, Bennett JE, Dolin R (eds). Churchill Livingstone Inc, New York. p346354.

52.- Nagl M, Neher C, Hager J, Pfausler B, Schmutzhard E, Allerberger F. Bactericidal activity of vancomycin in cerebrospinal fluid. Antimicrob Agents Chemother 1999; 43: 1932-4.

53.- Beenen LF, Touw D J, Hekker T A, Haring D A. Pharmacokinetics of intraventricularly administered teicoplanin in Staphylococci ventriculitis. Pharm

\section{World Sci 2000; 22: 127-9}

54.- Wilson A P R, Grüneberg R N. Chemistry, mode of action and antibacterial activity. En: Teicoplanin, the first decade. The Medicine Group (Education) Ltd. United Kingdom, 1997; 11-32.

55.- Bayston R, Hart C A, Barnicoat M. Intraventricular vancomycin in the treatment of ventriculitis associated with cerebrospinal fluid shunting and drainage. J Neurol Neurosurg Psychiatry 1987; 50: 1419-23.

56.- Pfausler B, Haring H P, Kampfl A, Wissel J, Schober M, Schmutzhard E. Cerebrospinal fluid (CSF) pharmakinetics of intraventricular vancomycin in patients with staphylococcal ventriculitis associated with external CSF drainage. Clin Infect Dis 1997; 25: 733-5.

57.- Leenders A C, Reiss P, Portegies P et al. Liposomal amphotericin B (amBisome) compared with amphotericin B both followed by oral fluconazole in the treatment of AIDS-associated cryptococcal meningitis. AIDS 1997; 11: 1463-71.

58.- Menichetti F, Fiorio M, Tosti A et al. High-dose fluconazole therapy for cryptococcal meningitis in patients with AIDS. Clin Infect Dis 1996; 22: 83840.

59.- Schwartz S, Milatovic D, Thiel E. Successful treatment of cerebral aspergillosis with a novel triazole (voriconazole) in a patient with acute leukaemia. Br J Haematol 1997; 97: 663-5.

60.- Wright P F, Kaiser A B, Bowman C M, McKee K T Jr, Trujillo H, McGee Z A. The pharmacokinetics and efficacy of an aminoglycoside administered into the cerebral ventricles in neonates: Implications for further evaluation of this route of therapy in meningitis. J Infect Dis 1981; 143: 141-7.

61.- Donlan R M. Biofilms and device-associated infections. Emerg Infect Dis 2001; 7: 277-81.

62.- Chiou C C, Wong T T, Lin H H et al. Fungal infection of ventriculoperitoneal shunts in children. Clin Infect Dis 1994; 19: 1049-53.

63.- Kaiser A B, Wright P F, McGee Z A, Dupont W D. Intraventricular gentamicin in meningitis (letter). Lancet 1980; II: 252.

64.- Fernández-Viladrich P, Corbella X, Corral L, Tubau F, Mateu A. Successful treatment of ventriculitis due to carbapenem-resistant Acinetobacter baumannii with intraventricular colistin sulfomethate sodium. Clin Infect Dis 1999; 28: 916-7.

65.- Alleyne C H Jr, Hassan M, Zabramski J M. The efficacy and cost of prophylactic and periprocedural antibiotics in patients with external ventricular drains. Neurosurgery 2000; 47: 1124-7.

66.- Prabhu V C, Kaufmann H H, Voelker J L et al. Prophylactic antibiotics with intracranial pressure monitors and external ventricular drains: a review of the evidence. Surg Neurol 1999; 52: 226-36.

Correspondencia a:

Alberto Fica Cubillos

E-mail: afica@ns.hospital.uchile.cl 